\title{
Response of colliding beam-beam system to harmonic excitation due to crab-cavity rf phase modulation
}

\author{
K. Ohmi,${ }^{1, *}$ R. Tomás, ${ }^{2, \dagger}$ Y. Funakoshi, ${ }^{1}$ R. Calaga,${ }^{3}$ T. Ieiri, ${ }^{1}$ Y. Morita, ${ }^{1}$ K. Nakanishi, ${ }^{1}$ \\ K. Oide, ${ }^{1}$ Y. Ohnishi, ${ }^{1}$ Y. Sun, ${ }^{2}$ M. Tobiyama, ${ }^{1}$ and F. Zimmermann ${ }^{2}$ \\ ${ }^{1}$ KEK, Tsukuba, Ibaraki 305-0801, Japan \\ ${ }^{2}$ CERN, CH 1211 Geneva 23, Switzerland \\ ${ }^{3}$ BNL, Upton, New York 11973, USA
}

(Received 20 August 2010; revised manuscript received 27 September 2011; published 28 November 2011)

During 2008 and 2009 dedicated beam experiments with crab cavities were performed in the KEKB. The goal was to measure the impact of crab-cavity radio frequency (rf) noise on the beam quality. These experiments were performed as a validation of the crab-cavity beam dynamics models in view of the possible use of crab cavities in the upgrade of the CERN Large Hadron Collider (LHC). An unexpected strong beam-beam instability was observed during the course of the experiments as a kind of frequency response. Understanding this finding required extensive multiparticle and single particle simulations plus an extra experimental session to consolidate the observations.

DOI: 10.1103/PhysRevSTAB.14.111003

PACS numbers: 29.20.db, 29.27.Bd

\section{INTRODUCTION}

The KEKB is an asymmetric electron-positron collider. The accelerator consists of a $3.5 \mathrm{GeV}$ positron storage ring (LER) and an $8 \mathrm{GeV}$ electron ring (HER) [1]. The electron and positron bunches cross at a $22 \mathrm{mrad}$ angle in the Belle detector. The KEKB has achieved a new world record luminosity $2.1 \times 10^{34} \mathrm{~cm}^{-2} \mathrm{~s}^{-1}$ in 2009 after the installation of one crab cavity per ring in 2007 . These cavities give a time dependent transverse deflection to the beams that results in head-on collisions at the interaction point (IP).

The LHC upgrade could incorporate crab cavities in order to maximize the luminosity [2]. However, unlike the KEKB, the LHC features a negligible synchrotron radiation damping. This calls for the use of accurate models of crab-cavity beam dynamics in order to validate an LHC upgrade based on crab cavities. Beam experiments at the KEKB were conducted in order to study two critical aspects, the impact of crab-cavity rf noise on the beam quality and techniques to measure the crab dispersion. Table I shows the machine parameters during the December 2008 crab-cavity experiments in the KEKB.

For several years crab-cavity rf phase noise has been a concern [3] since it results in off-center collisions at the IP. In the absence of radiation damping even small phase errors could cause a time-cumulative emittance blowup. In lepton machines, however, the excitation due to the phase error should reach a steady state thanks to the

\footnotetext{
*ohmi@post.kek.jp

†rogelio.tomas@cern.ch
}

Published by the American Physical Society under the terms of the Creative Commons Attribution 3.0 License. Further distribution of this work must maintain attribution to the author(s) and the published article's title, journal citation, and DOI. synchrotron radiation (at least for a small enough phase noise).

The phase noise of the KEKB crab cavities is dominated by a set of discrete frequencies [2]. Therefore it is justified to study the effect of phase noise at well-defined frequencies, rather than to assume white noise. In the following, "noise" refers to a signal with a single frequency component. Physically, response for harmonic excitation of the colliding beam-beam system is studied in this paper.

The beam-beam modes play important roles in this study. The beam-beam modes are defined by an eigensystem of the matrix product of revolution and linear beambeam kick,

$$
M=K M_{0},
$$

where

TABLE I. Machine parameters during the December 2008 crab-cavity experiments in the KEKB.

\begin{tabular}{lccc}
\hline \hline & Unit & HER & LER \\
\hline Particle & & $e^{-}$ & $e^{+}$ \\
Particles per bunch & $10^{10}$ & 4.1 & 6.3 \\
Number of bunches & & 100 & 100 \\
Horizontal emittance $\left(\epsilon_{x}\right)$ & $\mathrm{nm}$ & 24 & 18 \\
Horizontal tune $\left(Q_{x}\right)$ & & 44.507 & 45.512 \\
Vertical tune $\left(Q_{y}\right)$ & & 41.602 & 43.585 \\
Horizontal beam-beam parameter $\left(\xi_{x}\right)$ & & 0.100 & 0.111 \\
Vertical beam-beam parameter $\left(\xi_{y}\right)$ & & 0.077 & 0.099 \\
Synchrotron tune $\left(Q_{s}\right)$ & & 0.021 & 0.025 \\
Revolution frequency & $\mathrm{kHz}$ & 99.4 & 99.4 \\
Damping time & $\mathrm{ms}$ & 23 & 23 \\
Feedback damping time & $\mathrm{ms}$ & $\sim 1$ & $\sim 1$ \\
\hline \hline
\end{tabular}




$$
M_{0}=\left(\begin{array}{cccc}
\cos \mu_{e} & \sin \mu_{e} & 0 & 0 \\
-\sin \mu_{e} & \cos \mu_{e} & 0 & 0 \\
0 & 0 & \cos \mu_{p} & \sin \mu_{p} \\
0 & 0 & -\sin \mu_{p} & \cos \mu_{p}
\end{array}\right)
$$

and

$$
K=\left(\begin{array}{cccc}
1 & 0 & 0 & 0 \\
-4 \pi \xi_{e} & 1 & 4 \pi \xi_{e} & 0 \\
0 & 0 & 1 & 0 \\
4 \pi \xi_{p} & 0 & -4 \pi \xi_{p} & 1
\end{array}\right)
$$

where $\mu_{e(p)}=2 \pi Q_{e(p)}$ and $\xi_{e(p)}$ is the beam-beam parameter for electron (positron) beam. Eigenvalues of the colliding beam-beam system give $\sigma$ and $\pi$ modelike tunes in horizontal, $Q_{x, 1}=0.509$ and $Q_{x, 2}=0.564$, respectively. Note the $\pi$ mode tune is not $Q_{x}+\xi$, since the tunes are close to the half integer. Vertical eigenmodes are $Q_{y, 1}=$ 0.592 and $Q_{y, 2}=0.717$ for positron and electron dominant modes, respectively.

\section{CRAB-CAVITY RF PHASE NOISE}

During the beam experiments a sinusoidal phase modulation as a noise or a harmonic excitation was fed into the KEKB crab cavities while beams were in collision. The usual transverse feedback against multibunch instabilities [4,5] was working with a damping time of $\sim 1 \mathrm{~ms}$. Amplitude scans of the input phase modulation were performed for two modulation frequencies close to the horizontal and vertical tunes, respectively. Luminosity was recorded during these scans.

Multiparticle simulations have been performed for comparison with the experiments. The two beams, $e^{-}$and $e^{+}$, are represented by $2 \times 10^{5}$ particles each. A linear lattice transport is assumed. Transverse radiation damping (4000 turns) and feedback (100 turns of damping time) are included in the simulations with the same parameters as during the experiment. The beam-beam interaction is computed every turn according to the beam distributions [6].

Figures 1 and 2 show the luminosity versus the modulation amplitude of the HER and LER crab cavities, respectively. The frequency of the harmonic excitation in these two cases is set close to the horizontal tune of the machine. Results from the simulations described above are compared to the measurements showing an excellent agreement. The most striking feature observed both in measurements and simulations is the existence of a threshold in the excitation amplitude for the development of an instability causing the abrupt drop of luminosity. This threshold happens at an IP equivalent beam motion of about $4.5 \mu \mathrm{m}\left(\approx 0.03 \sigma_{x}^{*}\right.$ or $\approx 0.2$ degrees for the LER case). To compute the IP equivalent closed orbit distortion from the crab cavity (CC) phase modulation, the following equation is used:

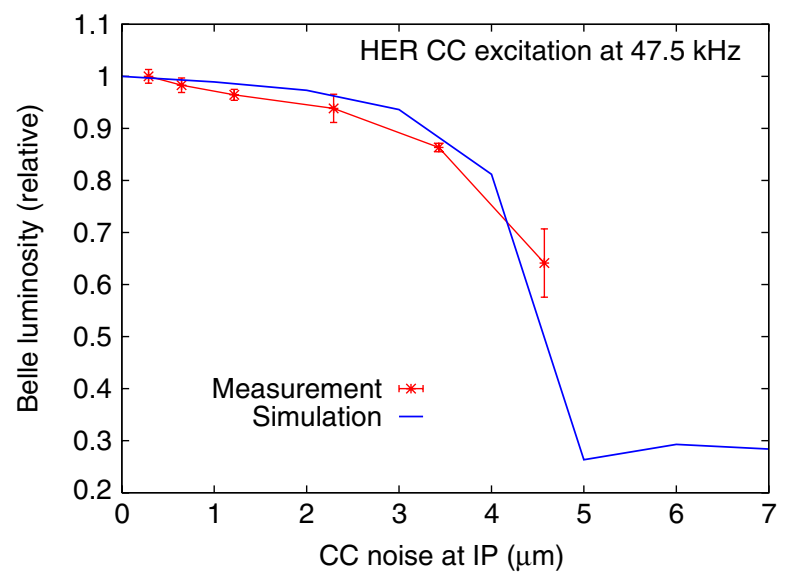

FIG. 1. Luminosity versus HER crab-cavity noise as extrapolated to IP displacement. The excitation frequency ( 0.522 in tune units) is close to the HER horizontal tune. The HER IP nominal horizontal beam size is $180 \mu \mathrm{m}$.

$$
x_{\mathrm{ex}} \approx \frac{c}{\omega} \tan \left(\frac{\theta}{2}\right) \phi_{\mathrm{ex}}
$$

where $\theta / 2$ denotes the half crossing angle (crabbing angle for each beam), $\omega$ is the angular frequency of the crab cavity, and $\phi_{\text {ex }}$ is the amplitude of the CC phase modulation. This equation can be simply derived from the geometrical constraints in the static case to provide head-on collisions at the IP and assuming small angles.

The abrupt luminosity loss is caused by an exponential rise of the horizontal emittance for amplitudes above the threshold. Figure 3 shows the simulated time evolution of the electron rms beam size for the four different noise amplitudes. The signature of an instability is clearly observed.

To understand this instability response, extensive simulations and analyses have been performed. Harmonic

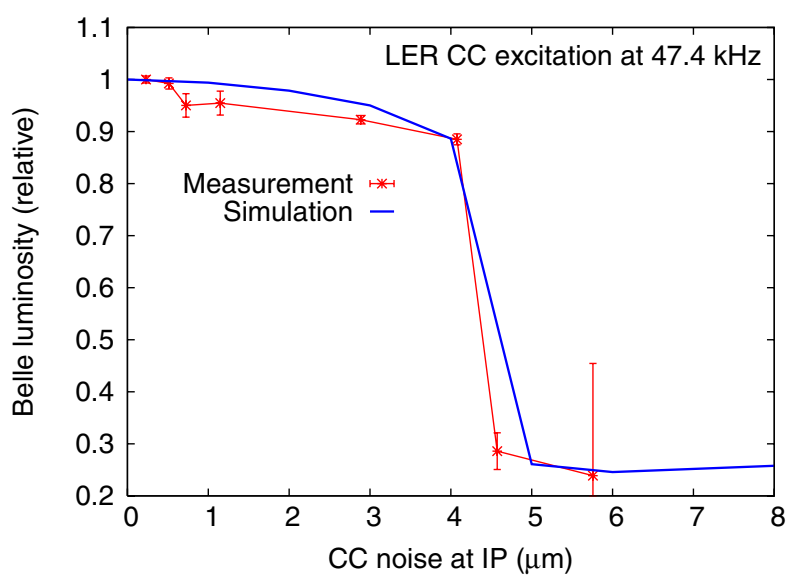

FIG. 2. Luminosity versus LER crab-cavity noise as extrapolated to IP displacement. The excitation frequency ( 0.523 in tune units) is close to the LER horizontal tune. The LER IP nominal horizontal beam size is $160 \mu \mathrm{m}$. 


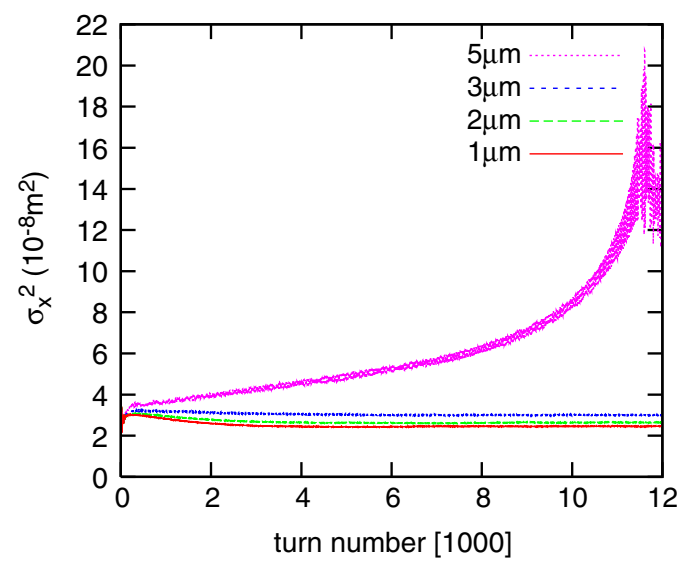

FIG. 3. Simulated rms beam size of the electron beam distribution versus turn number for different noise amplitudes, clearly revealing the existence of a threshold for the onset of the instability.

excitation corresponding to the crab-cavity noise is applied to positron beam with frequencies ranging between $0.497 f_{0}$ and $0.387 f_{0}$ in steps of $0.01 f_{0}$, where $f_{0}=$ $99.4 \mathrm{kHz}$ is the revolution frequency. The excitation amplitudes are $0.3,1.0,2.0,3.0,5.0,7.0$, and $10.0 \mu \mathrm{m}$ at the collision point. Figure 4 shows the Fourier spectra of the horizontal motion for the 11 different excitation frequencies at an amplitude of $1 \mu \mathrm{m}$. The peak tune of each line corresponds to the frequency of the harmonic excitation. The $\sigma$ and $\pi$ modes are located at 0.509 and 0.570, respectively, with the continuum spectrum $[7,8]$ in between these modes. The $\sigma$ mode features in-phase oscillations of the two beams at the IP. On the contrary, the $\pi$ mode is characterized by opposite-phase oscillations of the two beams at the IP.

Figure 5 shows the luminosity versus noise frequency for the different excitation amplitudes. This scan reveals

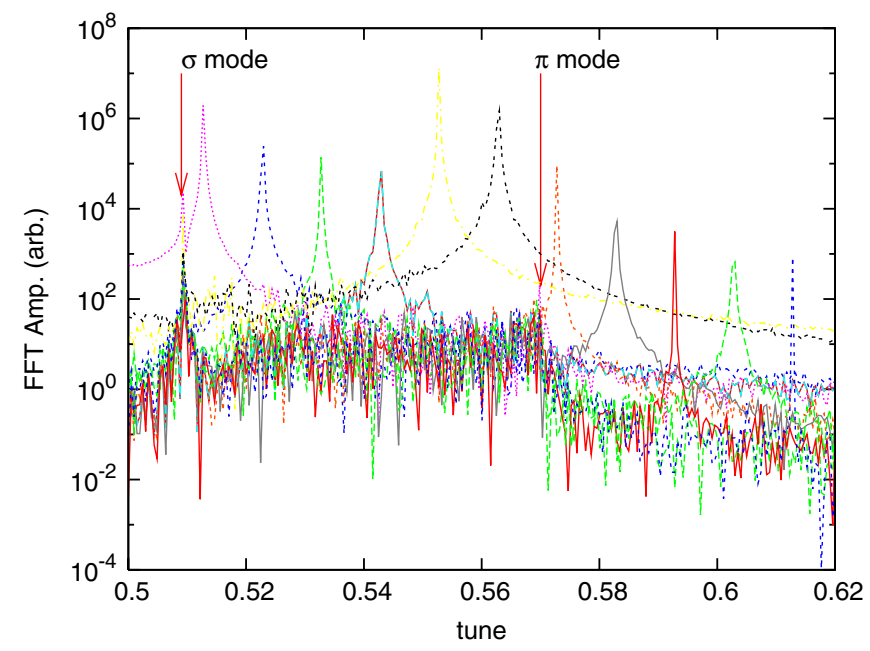

FIG. 4. Illustration of horizontal spectra of the centroid motion for the different 11 excitation frequencies. The IP equivalent excitation amplitude is $1 \mu \mathrm{m}$ for all excitation frequencies.

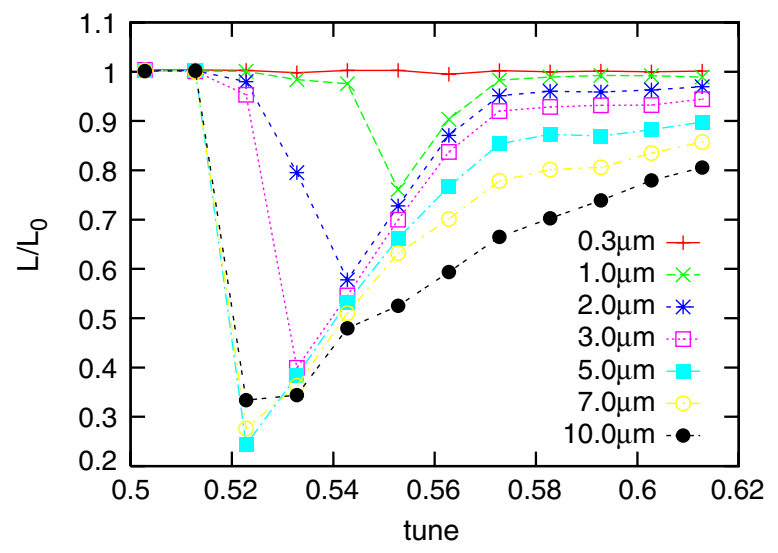

FIG. 5. Simulated relative luminosity versus frequency of the various excitation amplitudes.

that the instability is the strongest at a frequency of 0.523 for an IP equivalent amplitude of $\geq 5 \mu \mathrm{m}$. The abrupt luminosity losses are seen always between $\sigma$ and $\pi$ modes. Weaker luminosity losses are seen for lower amplitude at the noise frequency closer to linear $\pi$ mode. Exciting at the frequency closer to the $\sigma$ mode $(\sim 0.51)$ at these amplitudes does not cause significant luminosity loss.

Maximum Fourier amplitudes of the dipole motion are plotted versus excitation frequency for different amplitudes in Fig. 6. The behavior is similar to the luminosity seen in Fig. 5, but an enhancement at $\sigma$ mode $(0.51)$ is also seen.

Fourier analysis of $x_{p}-x_{e}$ and $x_{p}+x_{e}$ gives the frequency response of $\pi$ - and $\sigma$-modes amplitude. Figure 7 shows the $\pi$-mode (top) and the $\sigma$-mode (bottom) amplitudes versus excitation frequency for every amplitude. The behavior of the $\pi$ mode is similar to that of the luminosity, while $\sigma$ mode is enhanced when the excitation frequency is close to it without causing any luminosity loss. Therefore we find that the luminosity loss is caused by horizontal coherent oscillation of the beams in $\pi$ mode.

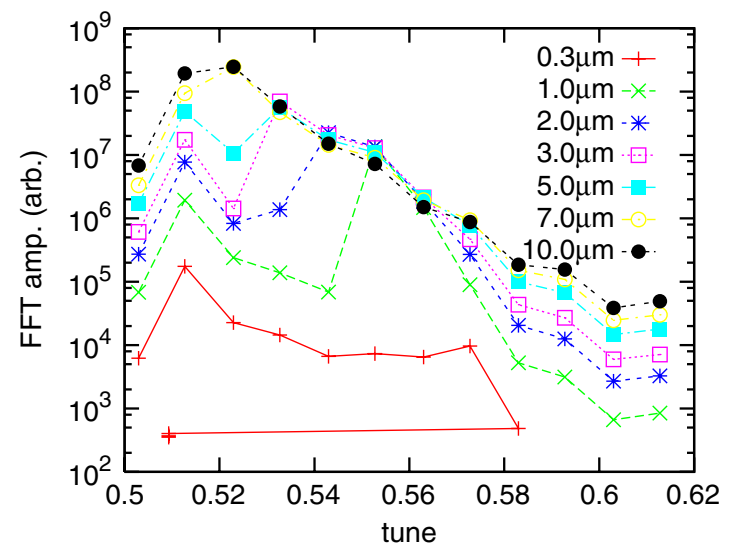

FIG. 6. Horizontal amplitude response of the positron beam to the harmonic excitation frequency and amplitude. 

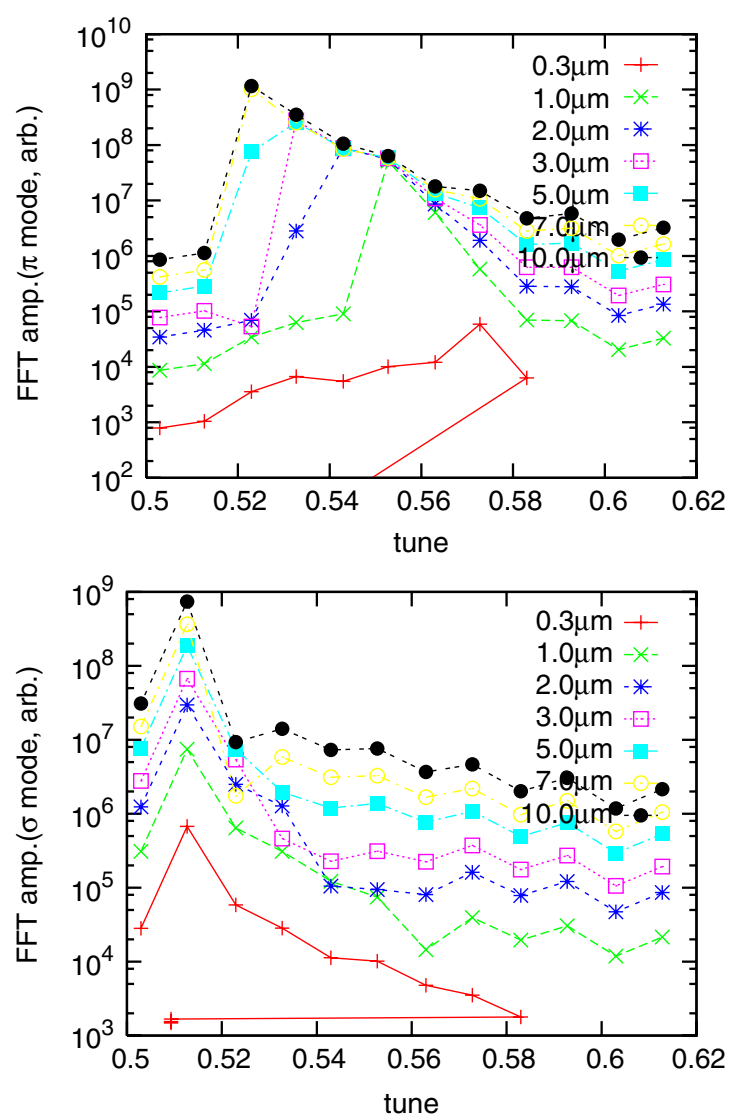

FIG. 7. Response of $\pi$ and $\sigma$ mode amplitudes for the harmonic excitation frequency and amplitude.

Figure 8 shows the time development of the instability displaying the electron and positron oscillation amplitude and phase difference versus turn number. These have been obtained by performing an fast-Fourier-transform (FFT)

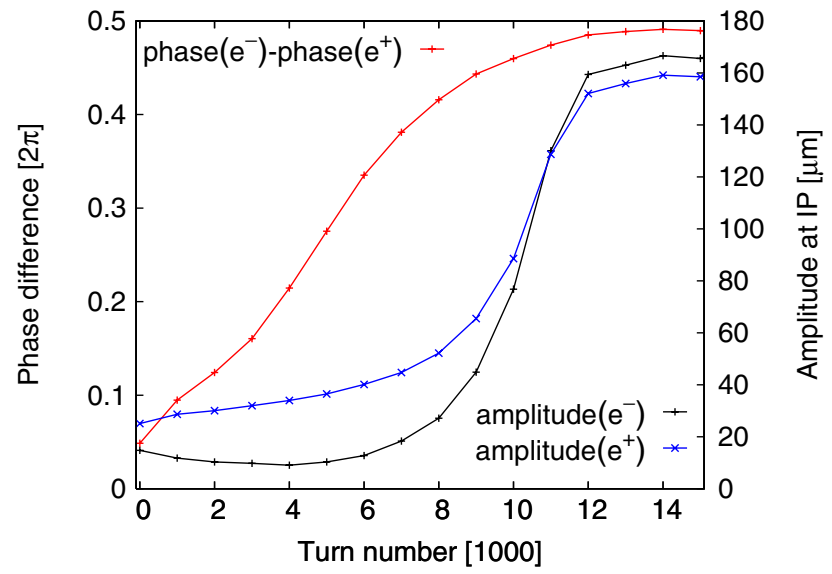

FIG. 8. Simulated time evolution of the driven spectral lines of the horizontal motion of the electron and the positron beams for an unstable case (CC noise at IP is $5 \mu \mathrm{m}$ with frequency 0.523 ). The phase difference between the driven spectral lines of the two beams is shown versus turn number. The amplitudes of the $e^{-}$and $e^{+}$motion at the same frequency are also shown. every 1000 turns over 1000 turns. The electron and positron beams start oscillating in phase (phase difference is very close to zero at 0 turns) at the driven frequency of 0.523 , however a monotonic increase in the phase difference versus time brings the beams to oscillate in opposite phases ( $\pi$ radians) after 9000 turns. The coherent oscillation amplitudes exponentially grow to values larger than the beam size $\left(\sigma_{x} \approx 100 \mu \mathrm{m}\right.$ including the beam-beam dynamic focusing). The instability saturates at about 12000 turns as shown in Figs. 3 and 8.

Figure 9 shows the cosine of the betatron phase difference between positron and electron beam, where the phase difference is calculated by $\cos \phi=\left\langle x_{e} x_{p}\right\rangle / \sqrt{\left\langle x_{e}^{2}\right\rangle\left\langle x_{p}^{2}\right\rangle}$ averaging after 9000 turns. The phase difference is $\pi$ for high frequency excitation. It abruptly jumps to 0 at lower frequency. The frequency jump to 0 depends on the excitation amplitude, and correlates to the jump of $\pi$ mode amplitude and luminosity loss. In a small amplitude, $\pi$ mode is induced at around the ordinary $\pi$ mode tune. A large amplitude $\pi$ mode is induced near the $\sigma$ mode tune causing a large luminosity degradation.

Once coherent oscillation arises, emittance growth is unavoidable due to the strong nonlinear force (smearing). The beam size response of positron beam is seen in Fig. 10. They are similar in behavior to the luminosity and dipole oscillation, so we can say that the emittance growth is basically due to the coherent motion.

The coherent oscillations of the electron and positron beams with exactly opposite phases cause off-centered collisions and, consequently, the luminosity loss. The dephasing of the driven oscillations towards $\pi$ radians seems to be the key ingredient of the instability since it is not observed in the stable cases. This motivates the following conjecture to explain the instability: Driving large beam oscillations lowers the $\pi$-mode frequency due to the nonlinear beam-beam interaction. Therefore when exciting between the $\sigma$ and the $\pi$ modes there exists an amplitude for which the driving frequency and the $\pi$ mode are in resonance. A similar phenomenon had been studied in

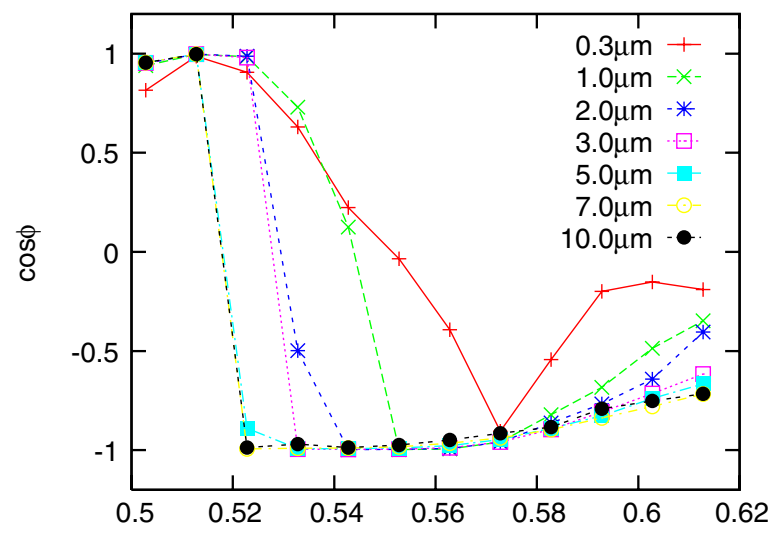

FIG. 9. Betatron phase difference between positron and electron beams for the excitation frequency and amplitude. 


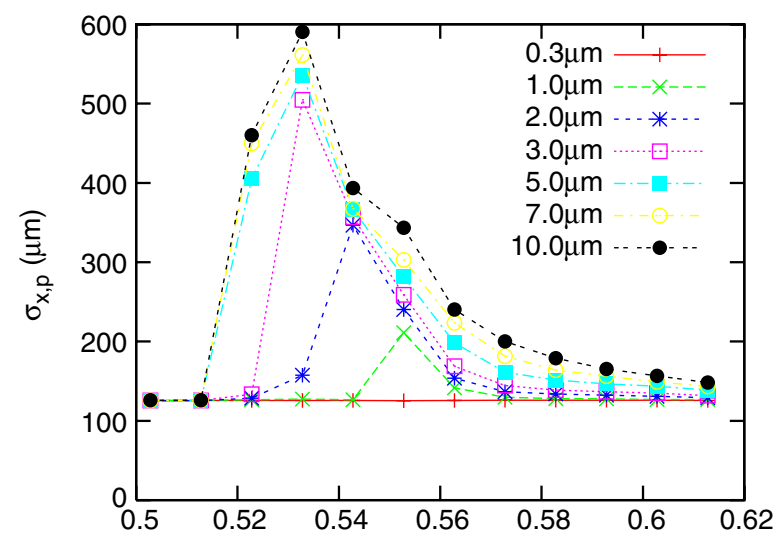

FIG. 10. Response of positron beam size for the harmonic excitation frequency and amplitude.

amplitude response of beam motion by Ieiri and Hirata [9] in TRISTAN.

This conjecture is illustrated by computing the eigenfrequencies of the beam motion in the presence of the beam-beam interaction for different oscillation amplitudes. Because of the nonlinearity of beam-beam interaction numerical simulations must be performed. The electron and positron bunches are represented by a single macroparticle to avoid filamentation. The beam-beam kick corresponds to that of a Gaussian bunch with constant beam size. A linear one-turn map is applied to transport the macroparticles around the accelerator. Free oscillations are simulated by giving an initial offset to the macroparticles. Figure 11 shows the results of these simulations. Both top and bottom plots show that for oscillations in the order of 2.5 beam sigma the frequency of the $\pi$ mode is reduced to 0.523 as would be required for the resonance condition of Figs. 7 and 8. The consistency between the observations, the multiparticle simulations, and the single macroparticle simulations validate, at least at a qualitative level, the previous conjecture for the mechanism of the instability.

Harmonically driven oscillations are the fundamental reason for the onset of the instabilities. During the 2008 experiments only one of the predicted instabilities was observed (the strong one at frequency of 0.52) and a direct observation of the driven oscillations could not be done for scheduling reasons. Therefore another dedicated experiment in December 2009 was done to search for the weaker instability or response (at a frequency of about 0.55 ) and to directly observe the driven oscillations by using the bunch oscillations recorder (BOR) [4]. Figure 12 shows the relative luminosity versus noise frequency as measured during the 2009 experiments, together with model predictions. The measured points at the noise amplitude of $0.4 \mu \mathrm{m}$ clearly reveal the existence of the predicted weaker instability at the frequency of 0.55 . The model prediction at $0.4 \mu \mathrm{m}$ shows the loss peak slightly displaced (at 0.56 ) and with half the amplitude of the measurement. The data for
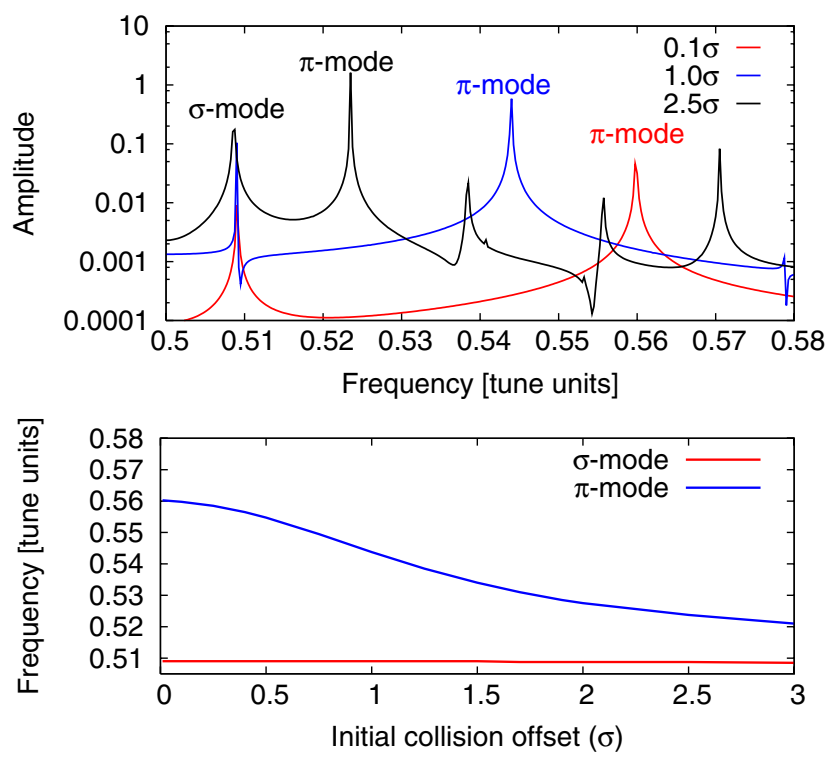

FIG. 11. Illustration of the dependence of beam-beam eigenmodes on the beam oscillation amplitude using a rigid bunch model. Top: horizontal spectrum for three cases with increasing initial collision offset showing a reduction of the $\pi$-mode frequency. Other spectral lines generated by the nonlinearity of the beam-beam interaction are observed for the $2.5 \sigma$ and $1.0 \sigma$ cases. Bottom: $\pi$ - and $\sigma$-mode frequencies versus initial collision offset.

the amplitude of $0.7 \mu \mathrm{m}$ is not dense enough to locate the loss peak and shows a larger loss than the model prediction at $0.52,0.54$, and 0.58 . The small discrepancies between model and measurement at this lower level of losses might be attributed to the uncertainties on beam parameters or to the lack of some ingredient in the simulation. However, it is remarkable that the weaker instability at lower excitation amplitude was first predicted by simulations and then observed in measurements. Figure 13 shows the measured amplitude of the beam oscillations extrapolated to the IP

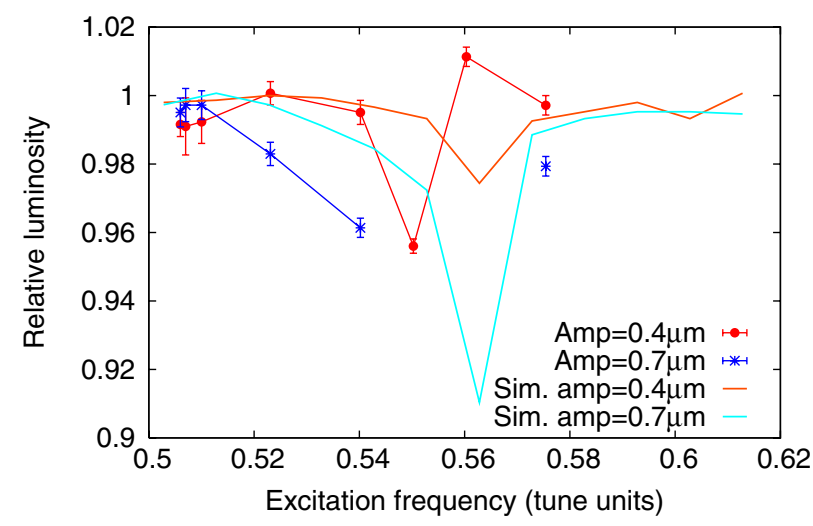

FIG. 12. Relative luminosity versus frequency of the harmonic excitation for two excitation amplitudes, both from measurements and simulations. The experimental points are connected with lines to guide the eye where considered appropriate. 


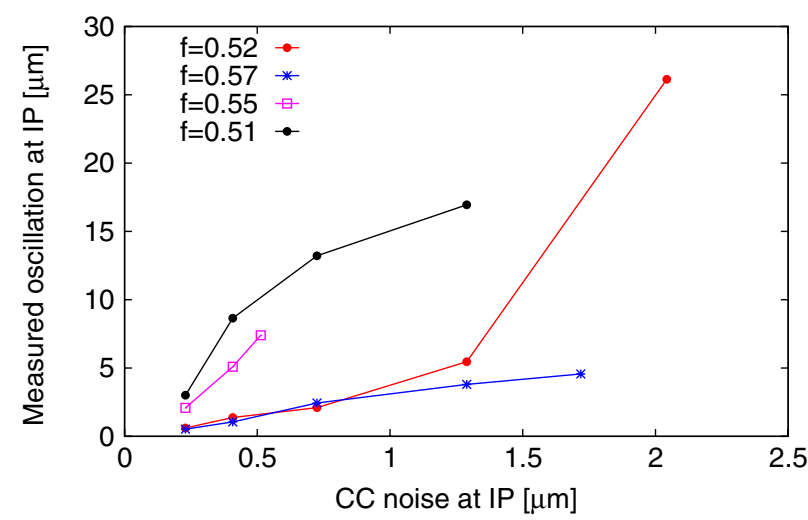

FIG. 13. Observed coherent oscillations versus amplitude of the $\mathrm{CC}$ harmonic excitation. Both quantities expressed as equivalents at the IP location. The nominal IP horizontal beam size is $160 \mu \mathrm{m}$.

versus the crab-cavity noise amplitude (as expressed in IP equivalent amplitude). The main goal of these data is to confirm the existence of coherent oscillations driven by the $\mathrm{CC}$ noise. The order of magnitude of these oscillations is comparable to that predicted by the numerical simulations in Fig. 8 taking into account that the maximum equivalent amplitude in the BOR measurements is $26 \mu \mathrm{m}$ for CC noise of $2 \mu \mathrm{m}$.

Amplitude scans were also performed for frequencies close to the vertical tunes, Figs. 14 and 15 . The crab-cavity deflects in the horizontal plane, therefore in the absence of transverse coupling no resonance effect is expected. Measurement and simulation agree in confirming the absence of any instability in the range shown on the plots. However, the agreement between measured and simulated luminosity loss is worse than when exciting close to the horizontal tune. This can only be explained by the lack of some ingredient in the simulation like transverse coupling.

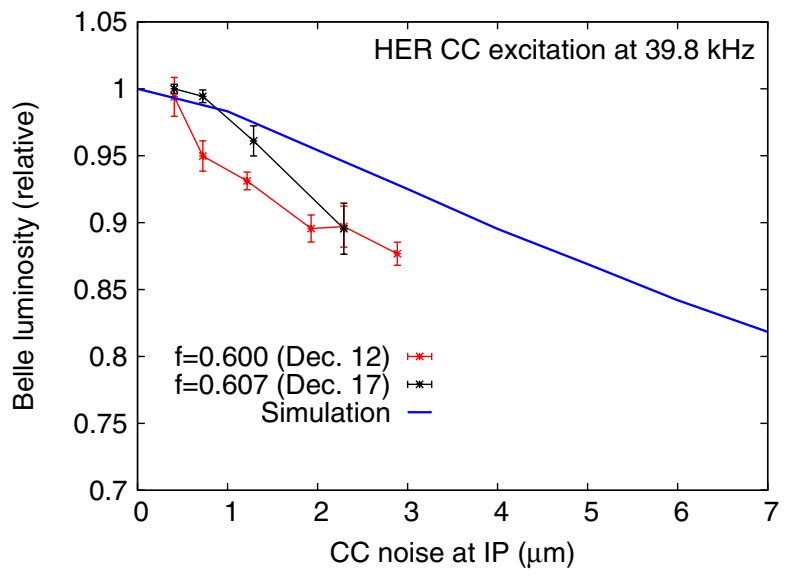

FIG. 14. Luminosity versus HER crab-cavity noise (harmonic excitation) as extrapolated to IP displacement. The excitation frequency (0.599 in tune units) is close to the HER vertical tune. The nominal IP horizontal beam size is $180 \mu \mathrm{m}$.

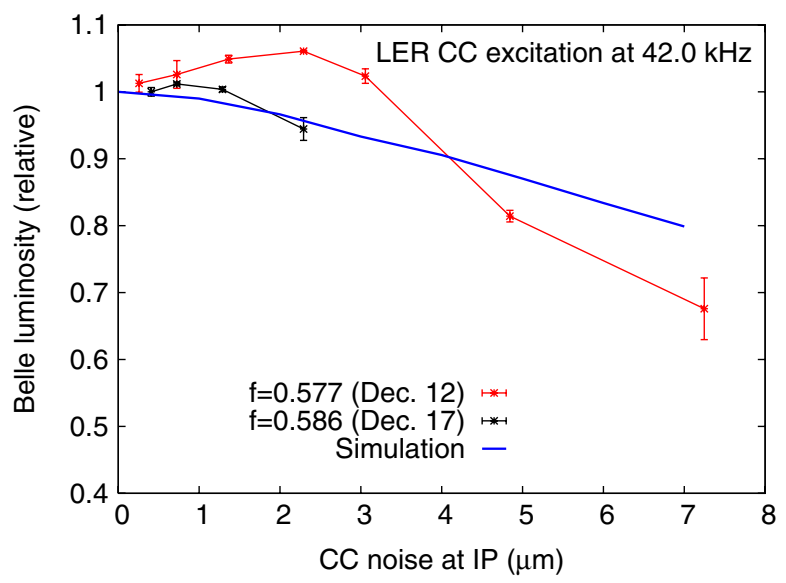

FIG. 15. Luminosity versus LER crab-cavity noise (harmonic excitation) as extrapolated to IP displacement. The excitation frequency ( 0.577 in tune units) is close to the LER vertical tune. The nominal IP horizontal beam size is $160 \mu \mathrm{m}$.

The LER measurement, in Fig. 15, is the most astonishing showing an increase of the luminosity for the small excitation amplitudes.

\section{CONCLUSIONS}

Beam dynamics experiments with crab cavities in the KEKB during 2008 and 2009 have served to verify the theoretical models and to discover an instability induced by the harmonic excitation in the presence of the beambeam interaction. This instability has been understood as a resonant excitation of the $\pi$ mode which exhibits an amplitude dependent frequency. Therefore for certain excitation frequencies there exists an amplitude threshold for which a resonant forced oscillation occurs.

The instability has been observed at two different frequencies and phase noise amplitudes both in measurements and simulations. The threshold for the onset of the strongest instability (when frequency is close to the machine tune) is found to be at an IP amplitude of about $4.5 \mu \mathrm{m}$ $\left(\approx 0.03 \sigma_{x}\right.$ for LER). The weaker instability between $0.55-0.56$ (close to the $\pi$ mode) develops at $0.4 \mu \mathrm{m}$ $\left(\approx 0.003 \sigma_{x}\right)$. This noise amplitude corresponds to 0.02 degrees of the $\mathrm{CC}$ phase noise.

The systematic study using a strong-strong beam-beam simulation explains the mechanism of the luminosity instability. The $\pi$ mode features an amplitude dependent frequency. Its frequency shifts towards the $\sigma$ mode for larger amplitudes. Therefore large oscillations require an excitation frequency closely above the $\sigma$ mode, while weak oscillations require an excitation frequency slightly below the small amplitude $\pi$-mode frequency.

These measurements set the first steps towards the validation of crab cavities in the LHC. On one hand, the proton beam in the LHC will not benefit from the damping by synchrotron radiation but, on the other hand, the beam-beam 
tuneshift of the LHC will not reach the current KEKB beambeam tuneshift. Dedicated LHC simulations using the beam dynamics models for CCs verified against KEKB beam measurements should shed light on the phase noise tolerances. Given the above results, it is advisable to aim at noise levels well below the observed 0.02 degrees for the onset of the weaker instability.

\section{ACKNOWLEDGMENTS}

R. Miyamoto provided a first version of the rigid bunch tracking code used in this paper. Thanks to M. Giovannozzi for proofreading this manuscript. This work is supported by the Large Scale Simulation Program No. 10-16 (FY2010) of High Energy Accelerator Research Organization (KEK). This work is also supported by the European Commission under the FP7 Research Infrastructures project EuCARD, Grant Agreement No. 227579.

[1] S. Kurokawa et al., KEK Report No. 95-7, 1995; Y. Funakoshi et al., in Proceedings of the 11th European
Particle Accelerator Conference, Genoa, 2008 (EPS-AG, Genoa, Italy, 2008), p. 1893.

[2] R. Calaga, U. Dorda, R. Tomas, F. Zimmermann, K. Akai, K. Ohmi, and K. Oide, in Proceedings of the 2007 Particle Accelerator Conference, Albuquerque, New Mexico (IEEE, New York, 2007), p. 1853.

[3] K. Ohmi et al., in Proceedings of the 2007 Particle Accelerator Conference, Albuquerque, New Mexico (Ref. [2]), p. 1496.

[4] M. Tobiyama and E. Kikutani, Phys. Rev. ST Accel. Beams 3, 012801 (2000).

[5] M. Arinaga, J. Flanagan, S. Hiramatsu, T. Ieiri, H. Ikeda, H. Ishii, E. Kikutani, T. Mimashi, T. Mitsuhashi, H. Mizuno, K. Mori, M. Tejima, and M. Tobiyama, Nucl. Instrum. Methods Phys. Res., Sect. A 499, 100 (2003).

[6] K. Ohmi et al., Phys. Rev. ST Accel. Beams 7, 104401 (2004).

[7] K. Yokoya and H. Koiso, Particle Accelerators (1990), Vol. 27, pp. 181-186.

[8] Y.I. Alexahin, in Proceedings of LHC'99 Workshop, Geneva, 1999 (Report No. CERN-SL-99-039 AP), p. 41.

[9] T. Ieiri and K. Hirata, in Proceedings of the 1989 Particle Accelerator Conference, Chicago, IL (IEEE, New York, 1989), p. 926. 